\title{
¿Tiene cabida en la empresa el desarrollo humano integral?
}

\section{Domènec Melé}

Resumen: El desarrollo humano integral -desarrollo de todo el hombre y de todos los hombres- abarca la vida entera $y$, por tanto, no queda excluido de la empresa. El desarrollo humano en la empresa tiene lugar por el trabajo y la convivencia dentro de la organización empresarial. Mejorar tal desarrollo es, en primer lugar, responsabilidad de cada persona, pero las condiciones de trabajo pueden favorecerlo o dificultarlo. En este contexto, los directivos de empresa, en primer lugar, tienen la responsabilidad, de no impedirlo ya sea mandando o motivando de modo contrario al verdadero desarrollo humano. Cumplido este requisito, la segunda responsabilidad es favorecerlo, de modo que se estimule el sentido de servicio y la adquisición de virtudes humanas. Esto último está relacionado con la organización del trabajo, la convivencia y la cultura organizativa y el modo de liderar.

Palabras Clave: Desarrollo humano integral, empresa, gerencia de empresas, condiciones de trabajo.

Fecha de recepción: 28-30 de junio de $2017^{2}$.

Fecha de admisión definitiva: 21 de junio de 2018 .

Does integral human development have a place in the company?

Abstract: The Integral human development, -development of the whole man and of all
Le développement humain intégral a-t-il une place dans l'entreprise?

Résumé: Le développement humain intégral -développement complet de I'homme et

1 Titular de la Cátedra de Ética Empresarial. IESE Business School, Universidad de Navarra.

${ }^{2}$ Fecha de celebración del Simposio. 
men- includes the whole life and, therefore, it does not remain excluded from the enterprise. Human development in the enterprise takes place through the work and the living together inside the managerial organization. To enhance such development is, in the first place, responsibility of each person, but the work-conditions may favor it, or make it more difficult. In this context, the enterprise's leaders, firstly, have the responsibility not to hinder it, be It ordering, or motivating in a contrary direction to the true human development. Once this requirement is accomplished, the second responsibility is to favor it, in such a way that the sense of service and the acquisition of human virtues may be stimulated. This last point is related to the labor-organization, the living together, and the organizational culture and the way of leading.

Key words: Integral human development, enterprise, enterprises management, work-conditions. de tous les hommes- implique la vie dans son ensemble, et donc, aussi le monde de l'entreprise. Le développement humain dans l'entreprise a lieu par le travail et par la cohabitation au sein de l'organisation de I'entreprise. Améliorer un tel développement est, d'abord, responsabilité de chaque personne, mais les conditions de travail peuvent le faciliter ou l'entraver. Dans ce contexte, la direction de l'entreprise est, en premier lieu, responsable de ne pas l'entraver que ce soit en permettant ou en motivant le contraire du véritable développement humain. Une fois cela fait, la deuxième responsabilité est de le favoriser de telle façon à ce que le sens du service et de l'acquisition des vertus humaines soient stimulés. Ceci est en rapport avec l'organisation du travail, la cohabitation, la culture organisatrice et la façon d'être un leader.

Mots clé: Développement humain intégral, entreprise, gestion d'entreprises, conditions de travail.

\section{Abreviaturas}

CA: Encíclica Centessimus annus, Juan Pablo II, 1991.

CDSI: Compendio de la doctrina social de la Iglesia, Pontificio Consejo Justicia y $\mathrm{Paz}, 2005$.

CIC: Catecismo de la Iglesia Católica, 1997.

CV: Encíclica Caritas in veritate, Benedicto XVI, 2009.

DHI: Desarrollo humano integral.

DSI: Doctrina social de la Iglesia.

GS: Constitución Gaudium et spes, Concilio Vaticano II, 1965.

HDI: Human Development Index.

LE: Encíclica Laborens exercens, Juan Pablo II, 1981.

LS: Encíclica Laudato si', Francisco, 2015.

PP: $\quad$ Encíclica Populorum progressio, Pablo VI, 1967.

QA: Encíclica Quadragesimo anno, Pío XI, 1931.

RN Encíclica Rerum novarum, León XIII, 1891.

SRS: Encíclica Sollicitudo rei socialis, Juan Pablo II, 1987. 


\section{Introducción}

El desarrollo de los empleados y directivos en la empresa, a menudo, es presentado por la literatura de dirección de empresas como una necesidad para la buena marcha de la empresa. Se suele argumentar que el éxito de una empresa depende en gran medida de las capacidades, hábitos y actitudes de las personas que la componen. Con frecuencia se escucha que los trabajadores -operarios y directivos- son el activo más valioso de una empresa y, de hecho, muchos abogan por captar, formar y retener personas con talento razonando que el medio más importante para avanzar y competir. Las empresas concretan la importancia del desarrollo profesional y personal en planes de desarrollo de empleados y carreras profesiones para directivos trabajan en la empresa.

Con el desarrollo profesional se busca potenciar sus capacidades, tenerles informados de los avances recientes en el sector y entrenarles en el uso de técnicas y medios de producción y distribución. En el desarrollo humano se ven ventajas en el modo de trabajar y relacionarse con otros, hacer frente a condiciones adversas o a situaciones imprevisibles. Estos enfoques, en último término, están orientados a mejorar el desempeño. Los costes que acarrean los planes de desarrollo se ven como una inversión que muy posiblemente se recuperará con creces.

Esta visión utilitaria del desarrollo de empleados y directivos contrasta con otra visión del desarrollo humana enfocado en la persona en sí misma, aunque sin descartar las ventajas que tal desarrollo conlleve para la eficacia empresarial o en cualquier otra actividad. La doctrina social de la Iglesia (DSI), aunque refiriéndose a un ámbito más general, se sitúa en esta última perspectiva, como veremos en el presente trabajo, abogando por el desarrollo humano de las personas y además con carácter "integral".

Más aún, la DSI - especialmente en la encíclica Populorum progressio (PP) de Pablo $\mathrm{VI}$ y con renovado énfasis en la Caritas in veritate (CV) de Benedicto XVI - insiste en invitar a todos a prodigarse para que se consolide cada vez con mayor firmeza una civilización orientada hacia la búsqueda de un desarrollo humano integral y solidario (cf. CPJP 2005, 3-4). ¿̇stá también la empresa incluida en este "todos" que ha de prodigarse en la búsqueda de tal desarrollo? A tenor de este texto se diría que sí, pero procurar este tipo de desarrollo no es algo generalmente aceptado en el mundo de la empresa.

Probablemente muchos coincidirían en afirmar que la familia y la escuela son instituciones adecuados para promover un desarrollo humano integral. Pero, $\dot{2}^{\text {tiene }}$ 
cabida tan desarrollo en la empresa? Y, si lo tiene, ¿cómo puede la empresa facilitar este desarrollo? ${ }_{2}$ Tiene en ello alguna responsabilidad los directivos de empresa?

El objetivo de esta comunicación es analizar estas cuestiones. Para ello, empezaremos por revisar el concepto de desarrollo humano y, más específicamente, la noción de desarrollo humano integral" (DHI) tal como lo presenta la doctrina social de la Iglesia. A continuación discutiremos en qué sentido el DHI tiene cabida en la empresa y quién tiene responsabilidad en tal desarrollo.

\section{2. ¿Qué se entiende por "desarrollo humano"?}

El término "desarrollo humano" tiene diversos significados, aunque con un fondo común. En un contexto empresarial, el desarrollo humano se ha definido como "una medición de logros de los seres humanos a través del avance del conocimiento, cambios biológicos, formación de hábitos $u$ otros criterios que muestran cambios con el tiempo." (Business Dictionary online) Está definición incluye, pues, elementos corporales (cambios biológicos) y mentales (conocimientos y hábitos), al tiempo abre la puerta a otros contenidos.

En el ámbito socio-político internacional el desarrollo humano se presenta, en contraste con el desarrollo económico, significando valoraciones colectivas de elementos considerados como componentes básicos de este desarrollo. En este sentido y desde hace algunos años, a los índices de desarrollo económico, medidos en términos de PIB y tradicionalmente utilizados de modo exclusivo para medir el desarrollo de un país, se han añadido el Índice de Desarrollo Humano (Human Development Index, HDI). Este índice incluye tres conceptos básicos: salud y longevidad de vida, grado de escolarización y nivel de vida.

El HID ha estimulado el debate sobre las prioridades de las políticas gubernamentales, más allá de lo puramente económico, mediante datos empíricos de condiciones externas. Presentar índices de desarrollo humano y no sólo económico ha sido un gran avance. Sin embargo, este índice tiene obvias limitaciones. Por una parte, es un dato "macro" de carácter estadístico que no se fija en el desarrollo de cada persona, ni pretende señalar en qué consiste tal desarrollo personal. Por otra parte, excluye factores que parecen significativos para definir el desarrollo humano de un país. Concretamente, no se refleja la pobreza, la seguridad ciudadana, las desigualdades y discriminaciones, y la participación en la vida social. 
Más completo es el enfoque de las capacidades de Amartya Sen, y los subsecuentes desarrollos de Martha Nussbaum y otros, que presentan diversas dimensiones del desarrollo humano, que no detallaremos aquí por razones de brevedad. Estos enfoques han sido utilizados en estudios sobre la pobreza, psicología intercultural, filosofía moral, indicadores de calidad de vida, desarrollo participativo y necesidades básicas. Las listas presentadas son significativas, sin embargo, con frecuencia se les acusa también de ser incompletas (Alkire 2002) y, en efecto, no parece que el desarrollo humano pueda reducirse a un listado cerrado de componentes.

En su sentido etimológico "desarrollo humano" significa crecimiento o despliegue de aquello que puede considerarse genuinamente humano. Un correcto entendimiento de desarrollo humano presupone que en lo humano hay inherente una teleología con unos fines a alcanzar que orientan el crecimiento. Supone también la existencia de algo específicamente humano que lo distingue de otros seres que permite un desarrollo humano.

Al menos desde Aristóteles, muchos aceptan que ese algo propio del ser humano es la racionalidad, aunque lo humano incluye también corporeidad. El hombre -afirmaba este filósofo- posee "cierta vida activa propia del ente que tiene razón" (Aristóteles 1999 [s. IV a. C], lb 1, cap. 7, 1098a), lo cual vulgarmente se ha traducido en la conocida definición del hombre como "animal racional", aunque más bien habría que decir: un animal que posee logos (razón). La razón es capacidad para indagar la realidad descubriendo la verdad que encierra y discurrir sobre ella, aunque este logos sea imperfecto y esté lejos de conocer toda la verdad del mundo e incluso de uno mismo. La razón tiene también cierta capacidad para descubrir la verdad de la acción, es decir, aquello que es bueno en algún aspecto particular (razón técnica): aquello que es bueno para construir una casa, por ejemplo, lo aquello otro que contribuye a la eficiencia de una organización), y también lo que es bueno en un sentido general (razón práctica): aquello que es lo más conveniente en un sentido absoluto porqué contribuye a mejorar la calidad humana de quien lo realiza). La racionalidad en el comportamiento es, pues, una característica del ser humano que se perfecciona adquiriendo sabiduría, tanto para conocer la verdad (sabiduría) como para discernir el bien y el mal en orden a la acción (sabiduría práctica).

Otros autores sugieren que lo más específicamente humano es la libertad, que incluye auto-posesión de los propios actos y auto-determinación para decidir llevarlos a cabo; esto es, una voluntad libre no determinada por estímulos externos, aunque puede estar condicionada por ellos. La libertad así entendida, no excluye la razón, sino que la presupone y la utiliza para deliberar acerca de 
qué es lo más conveniente decidir y hacer $y$, en definitiva, para un correcto uso de la libertad. A su vez, la razón no excluye la voluntad libre sino que la incluye en su orientación a la acción. La voluntad se perfecciona por las virtudes, que la refuerzan para actuar bien.

Podría añadirse que también la esfera de la afectividad, con su rico abanico de emociones y sentimientos caracteriza lo humano, en la medida en que están conectados con el conocimiento racional y la voluntad libre. Así, la angustia ante el conocimiento de una enfermedad y la esperanza de que puede ser vencida, o el sufrimiento compasivo experimentado por el dolor de una persona querida, o la alegría por haber descubierto algo valioso. También algunos animales tienen reacciones que, por analogía, con los sentimientos humanos llamamos sentimientos, pero no surgen del conocimiento racional sino sensitivo.

Particularmente humano es el amor que va más allá de la posesión del otro (eros), manifestando donación de lo que uno es o posee al otro (agápe). Puede ir impregnado de un sentimiento más a menos intenso, pero no es mero sentimiento sino amor de la voluntad, guiado por la razón. También la corporalidad es humana y, en muchos aspectos, es consistente con el carácter racional y libre del ser humano y de su afectividad. Por ejemplo, el rostro que expresa pensamientos, emociones y sentimientos, y la mano configurada para llevar a cabo operaciones complejas derivadas de la razón técnica. Aunque ideologías materialistas niegan el carácter espiritual de la razón y la voluntad libre hay buenas razones para afirmar que la racionalidad y la voluntad libre son de naturaleza inmaterial, y por tanto espiritual, aunque utilicen una base orgánica para su ejercicio. Un recto desarrollo afectivo es también parte del desarrollo humano.

Razón, libertad y afectividad tiene un carácter teleológico, y es precisamente tal teleología lo que determina el desarrollo humano. La racionalidad está orientada al conocimiento de la verdad y la voluntad a hacer el bien (aquello que nos perfecciona), mientras que la afectividad se orienta a amar a quien descubre como digno de ser amado. El cuerpo humano tiene también una teleología, marcada en su desarrollo biológico que sigue leyes físicas, aunque ciertos aspectos de la corporeidad puede también desarrollarse por influencia de la voluntad (p. e., desarrollando habilidades manuales).

El florecimiento o desarrollo humano indica aquello que lleva a plenitud lo humana y que orienta el desarrollo. De acuerdo con lo dicho anteriormente está relacionado con las capacidades humanas superiores: conocer la verdad (razón) y amar y hacer el bien (voluntad y afectividad). Este conocimiento incluye reconocer el valor 
intrínseco de las personas aquello Trascendente que da sentido último a la vida humana. Para la fe cristiana ese Transcendente es un ser Personal que llamamos Dios: un ser que ama y es digno de ser amado y que, en un determinado momento histórico, se ha hecho hombre en Jesucristo.

En definitiva, el desarrollo humano consiste en mejorar el conocimiento profundo de Dios y de las personas humanas y la capacidad de tratarlas como corresponde, que se traduce en amar a Dios y a las demás personas como bien propio. Para ello son de gran relevancia la sabiduría y las virtudes, hábitos que contribuyen a la excelencia humana y que, de uno u otro modo, hacen posible la donación a los demás (agápe). Las virtudes fortalecen la razón y la voluntad y por ello contribuyen decisivamente al desarrollo humano.

El desarrollo humano es una necesidad radical del ser humano. Todos los seres vivos necesitan crecer y la teleología biológica lo lleva a cabo contando con los nutrientes que necesitan y que toman de su entorno natural. De otro modo, mueren o quedan en un estado raquítico diverso de lo que exige su naturaleza. Las personas necesitan también desarrollarse, pero no sólo en lo biológico. Racionalidad, libertad y afectividad exigen crecimiento en el conocimiento de la verdad y del bien, en la capacidad volitiva para actuar bien (virtudes) y en la calidad humana de los afectos. De otro modo, habría un crecimiento anquilosado o incluso una erosión en humanidad, como ocurre en las personas que actúan en la vida sin rumbo ni sentido, o con guías equivocadas, con malos hábitos o adicciones que limiten su capacidad de actuar bien.

A la necesidad radical de desarrollo humano le siguen otras necesidades exigidas por la condición humana: necesidades materiales (exigidas por la corporeidad), cognitivas (facilitadoras del acceso al conocimiento), operativas (promoción de capacidades técnicas y morales) y afectivas (facilitadoras del afecto recíproco con los demás).

\section{El concepto de "Desarrollo Humano Integral" en la DSI}

La DSI no se aleja de la concepción del desarrollo humano como crecimiento en el conocimiento de la verdad y en la capacidad para amar a Dios y a los demás, del cual participa en alguna medida la corporeidad.

En primer lugar, la DSI pone gran cuidado en diferenciar el desarrollo económico y el desarrollo humano, añadiendo que este último ha de ser integral. En este sentido, 
Pablo VI afirmaba: "El desarrollo no se reduce al simple crecimiento económico. Para ser auténtico, debe ser integral, es decir, promover a todos los hombres y a todo el hombre." (PP 14). Una definición adoptada por Benedicto XVI, para quien desarrollo humano integral es también "desarrollo de todo el hombre y de todos los hombres" (CV 8, 18) o, en otras palabras, el desarrollo de las personas y de los pueblos (cf. CV 52, 53, 54).

Dos elementos concurren en la idea de "integral". Uno es que se refiera a "todos" los hombres, sin exclusión alguna, y de "todo el hombre", es decir del hombre completo. Vamos a detenernos en ellos, empezando por éste último.

\section{I. Desarrollo de "todo el hombre"}

En relación a "todo el hombre", desarrollo humano integral debe tomar en consideración el carácter unitario del ser humano, que incluye corporalidad, capacidades psíquicas -como memoria, diversas formas de inteligencia-, así como las capacidades espirituales orientadas al conocimiento abstracto - razón-y al actuar guiado por la razón, auto-poseído y auto-determinado - la voluntad.

El carácter unitario de la persona es parte esencial de la DSI: el hombre es un "todo"; "cuerpo y alma, corazón y conciencia, inteligencia y voluntad" (GS, 3); no una dualidad "cuerpo-mente", sino "una unidad de cuerpo y alma" (GS 14). Esta base antropológica es clave para el desarrollo humano integral de cada persona. Ese carácter unitario de la persona es subrayado por Benedicto XVI al señalar que "el auténtico desarrollo del hombre concierne de manera unitaria a la totalidad de la persona en todas sus dimensiones." (CV 11).

El desarrollo integral de cada persona incluye aspectos materiales, pero ha de considerar también, y muy especialmente, la naturaleza espiritual del hombre y su apertura a la transcendencia. Benedicto XVI se refiere al carácter espiritual de la persona y a la importancia del progreso espiritual como parte esencial del desarrollo.

El desarrollo debe abarcar, además de un progreso material, uno espiritual, porque el hombre es "uno en cuerpo y alma» (GS 14), nacido del amor creador de Dios y destinado a vivir eternamente (CV 76).

En este desarrollo el progreso espiritual es primordial.

La criatura humana, en cuanto de naturaleza espiritual, se realiza en las relaciones interpersonales. Cuanto más las vive de manera auténtica, tanto más madura también en la propia identidad personal. El hombre se valoriza no aislándose sino poniéndose en relación con los otros y con Dios (CV, 53). 
El ser humano -añade el Papa- se desarrolla cuando crece espiritualmente, cuando su alma se conoce a sí misma y la verdad que Dios ha impreso germinalmente en ella, cuando dialoga consigo mismo y con su Creador. Lejos de Dios, el hombre está inquieto y se hace frágil (CV, 76).

Se pone, pues, de relieve la importancia de buscar la verdad y de una recta orientación de la "relación" derivada de la condición personal del hombre (CV, 53) para un auténtico desarrollo humano. Además de la relación con Dios, la relación con los demás contribuye a la propia perfección en la medida en que implica donación. Como señala un celebrado texto del Concilio Vaticano II, el hombre "no puede encontrar su propia plenitud si no es en la entrega sincera de sí mismo a los demás." (GS 24). En efecto, el amor generoso a los demás, contribuye a la propia excelencia.

La importancia de lo espiritual en el desarrollo humano había ya sido puesta de relieve por Juan Pablo II al afirmar que

un desarrollo no solamente económico se mide y se orienta según esta realidad y vocación del hombre visto globalmente, es decir, según un propio parámetro interior. Este, ciertamente, necesita de los bienes creados y de los productos de la industria, enriquecida constantemente por el progreso científico y tecnológico. Y la disponibilidad siempre nueva de los bienes materiales, mientras satisface las necesidades, abre nuevos horizontes (...) Mas para alcanzar el verdadero desarrollo es necesario no perder de vista dicho parámetro, que está en la naturaleza específica del hombre, creado por Dios a su imagen y semejanza (SRS, 29).

En esta naturaleza específica del hombre se incluye la capacidad humana de deliberar y actuar con libertad, pero también los afectos y la corporeidad que, de algún modo, participan de la espiritualidad de la persona. En efecto, el cuerpo es humano precisamente porque está animado por el alma espiritual y por ello participa también de la condición de "imagen de Dios" (CIC, 364).

El desarrollo de aspectos particulares incluidos en el ser humano puede contribuir al desarrollo humano integral en la medida en que no impidan el crecimiento espiritual o aun lo fortalezcan. Así desarrollar la musculatura o adquirir pericia en la práctica de algún deporte puede contribuir al desarrollo humano integral, si no perjudica otros aspectos más valiosos y, en términos positivos, contribuye a un mejor ejercicio de la mente y de la voluntad. Tal puede ser el caso de muchos deportes -aunque no siempre- si contribuyen a un mejor ejercicio de la razón -mens sana in corpore sano, decían los antiguos- y fomenten buenos hábitos en la voluntad (cooperación con otros, generosidad, etc.). Aprender a tocar algún instrumento, suele ayudar a fomentar el sentido estético, aprender a manejar programas de 
ordenador, el sentido lógico y aprender a gestionar actividades de voluntariado, el sentido de servicio a los demás.

\subsection{El desarrollo de "todos los hombres"}

Mientras la expresión desarrollo de "todo el hombre", como hemos visto, entraña una exigencia personal, al hablar de "todos los hombres" se alude a un objetivo social: poner los medios para que el desarrollo de "todo el hombre" alcance a "todos los hombres". De modo más específico, el desarrollo humano de una comunidad abarca a todos quienes forman parte de ella. Estas comunidades pueden ser pequeñas, como la familia o una pequeña empresa, pero también alcanza a las grandes corporaciones, que son comunidades con vínculos internos y metas comunes. Hay también comunidades nacionales y supranacionales.

El desarrollo de las personas que forman una comunidad es bien común de la misma. La comunidad contribuye a este desarrollo mediante condiciones exteriores que lo facilitan. Estas condiciones afectan a elementos materiales, culturales, organizativos y morales. En un país es bien común, entre otros muchos elementos, el que se facilite el acceso a la vivienda, la educación y a la asistencia sanitaria; el que hay trabajo y medios para ganarse la vida, un medio ambiente limpio y saludable; que exista respeto por las personas y los derechos humanos, justicia y paz social, un gobierno responsable que trabaje por el bien de todos, un sistema jurídico y garantista y una cultura de solidaridad y cooperación.

Cabe pensar que también la empresa puede crear condiciones para el bien común $y$, en definitiva, favorecedoras del desarrollo de que quienes forman la comunidad-empresa. A Volveremos sobre este tema más adelante.

\section{4. ¿Desarrollo humano integral en la empresa?}

Como ha indicado en la introducción, en la empresa suele hablarse de "crecimiento personal" (personal growth) y desarrollo profesional (professional development), prestando atención a lo que resulta favorable para un buen desempeño empresarial. Se lleva a cabo con planes concretos y diversos estudios psicológicos tratan de medir algunos aspectos específicos de crecimiento personal, por ejemplo, la iniciativa (Robitschek et. al. 2012). 
También se ha prestado atención a la auto-realización o actualización de potencialidades de los empleados con un marcado carácter subjetivo. Entró en la literatura empresarial con el descubrimiento y valoración de necesidades en el ser humano (Maslow 1943, 1970) y con vistas a la motivación. En particular, Maslow habla de la necesidad de "auto-actualización" (self-actualization) definida por este autor como "El deseo de realización personal, es decir, la tendencia de que el individuo se realice en lo que es potencialmente" (the desire for self-fulfillment, namely the tendency for him [the individual] to become actualized in what he is potentially)" Maslow ve la auto-actualización como deseo para lograr lo que cada uno ambiciona más que como una llamada a desarrollar las capacidades que uno tiene como ser humano y como persona, que es lo propio del DHI propuesto por la DSI. También la psicología actual ve el "auto-actualización" o "auto-realización" como deseo para desarrollar potencialidades deseadas (Gleitman et al. 2004).

La voluntad subjetiva de auto-realización puede coincidir, o no, con el desarrollo humano en aquello que es perfeccionamiento o florecimiento humano, dependiendo de cada persona. La pregunta es si además de procurar crecimiento personal y profesional con vistas a mejorar la motivación y la eficacia, el desarrollo humano integral tiene también cabida en la empresa.

Para responder a esta cuestión, lo primero que conviene señalar es que el desarrollo humano de todo el hombre es un proceso vital y, por tanto, todas las facetas de la vida humana pueden contribuir, en un sentido o en otro, a tal proceso. Esto incluye la vida en la empresa $y$, en muchos casos, en mayor extensión que en otros ámbitos, dada la elevada proporción de tiempo de vida consciente que la mayoría de personas pasan en una empresa o en otros ámbitos profesionales en cierto modo asimilables a la empresa.

Hay aspectos del desarrollo personal cuyo ámbito más propio es la familia, la escuela o la comunidad religiosa, que la empresa. No obstante, sin ser tan amplio, el desarrollo en la empresa entraña cierto sentido comprensivo ya que incluye, como mínimo, desarrollo profesional -por lo menos, aprendido por la propia actividad y a veces también con educación externa- y diversos aspectos de desarrollo humano, generado por la convivencia empresarial y la voluntad personal de mejora. Pueden incluir, por ejemplo, aumento del sentido de cooperación, desarrollo de nexos relacionales, una mayor valoración del trabajo y mejora de la voluntad de servir a los demás.

El desarrollo humano en la empresa es "integral" en el sentido de que es "unitario", es decir, abarca a toda la persona. El desarrollo humano contribuye al 
desarrollo profesional, ya que este último no es una mera adquisición de técnicas y habilidades sino que también requiere hábitos humanos de gran relevancia profesional (responsabilidad, veracidad, fiabilidad, etc.). A su vez, el desarrollo profesión exige esfuerzo (concentración, estudio, diligencia, etc.) que contribuyen al desarrollo humano. En otras palabras, el desarrollo humano y el desarrollo profesional se integran y se complementan mutuamente. Esta influencia recíproca ha sido ya advertida por algunos al señalar que el desarrollo personal (aunque medido en sentido subjetivo) tiene una influencia en el desarrollo profesional, especialmente en la sociedad del conocimiento y viceversa (Hafer and Sirgy 1983; Wacowska 2014).

La respuesta a la cuestión formulada inicialmente de si el desarrollo humano integral tiene o no cabida en la empresa, debe ser afirmativa. El desarrollo humano integral es posible en la empresa si hay una voluntad por parte de cada persona y no hay factores organizacionales suficientemente fuertes que lo impidan. Más aún, como veremos más abajo, la empresa puede favorecerlo.

\section{Desarrollo humano integral en la empresa: responsabilidad personal e institucional}

La DSI presenta el desarrollo humano integral como una llamada a cada persona y responder a esta llamada incumbe a cada uno. En otras palabras, cada persona tiene la responsabilidad de crecer en humanidad -en aquello que le caracteriza como ser humano-. En efecto, como escribe Benedicto XVI,

todos los hombres perciben el impulso interior de amar de manera auténtica; amor y verdad nunca los abandonan completamente, porque son la vocación que Dios ha puesto en el corazón y en la mente de cada ser humano (CV 1).

Esta intuición humana es perfeccionada desde la fe:

Jesucristo purifica y libera de nuestras limitaciones humanas la búsqueda del amor y la verdad, y nos desvela plenamente la iniciativa de amor y el proyecto de vida verdadera que Dios ha preparado para nosotros (CV 1).

La DSI pone el acento en las personas más que en las instituciones en el logro del $\mathrm{DHI}$, aunque sin desmerecer la influencia de las instituciones. De modo inequívoco, Benedicto XVI refiriéndose al DHI afirma: "ninguna estructura puede garantizar dicho desarrollo desde fuera y por encima de la responsabilidad humana" (CV 17). En otro lugar hace notar que a lo largo de la historia se ha creído con frecuencia que 
la creación de instituciones bastaba para garantizar a la humanidad el ejercicio del derecho al desarrollo, depositado una confianza excesiva en dichas instituciones, casi como si ellas pudieran conseguir el objetivo deseado de manera automática.

En realidad-añade-, las instituciones por sí solas no bastan, porque el desarrollo humano integral es ante todo vocación y, por tanto, comporta que se asuman libre y solidariamente responsabilidades por parte de todos (CV 11).

La primacía de la persona no excluye las instituciones que juegan también un gran papel en el DHI. Dicho esto, conviene que nos fijemos en la empresa como institución social, la cual pueden contribuir en sentido positivo o negativo al DHI de las personas que la integran. El desarrollo de las personas involucradas en la empresa puede considerarse parte de la finalidad de la empresa, al menos si aceptamos que

finalidad de la empresa no es simplemente la producción de beneficios, sino más bien la existencia misma de la empresa como comunidad de hombres que, de diversas maneras, buscan la satisfacción de sus necesidades fundamentales y constituyen un grupo particular al servicio de la sociedad entera (CA 35).

El desarrollo humano integral, como hemos visto, es una necesidad fundamental y, por tanto, no debe ser excluido de la finalidad de la empresa en relación con las personas involucradas en ella.

Desde esta perspectiva, surgen dos exigencias de justicia por parte la empresa en relación con el $\mathrm{DHI}$ de quienes forman parte de ella. La primera y más elemental es no impedir tal desarrollo; la segunda, fomentarlo. De ello nos ocuparemos a continuación.

\section{I. No impedir el DHI}

Las personas tiene el deber de desarrollarse y el correspondiente derecho a que se respeta tal deber. Por tanto, no impedir el desarrollo humano integral es una exigencia de justicia

El desarrollo humano en la empresa se puede impedir con acciones mandatarias o persuasivas que corrompan las personas. Por ejemplo, involucrándolas en fraudes o sobornos o mediante presiones organizativas para obtener resultados "como sea", incluyendo actuaciones poco rectas. También con ejemplos negativos desde la dirección o fomentando valores negativos por parte de quienes lideran la empresa o mediante unas condiciones de trabajo en las cuales es muy difícil el desarrollo 
de virtudes. Otro modo de impedir el desarrollo es la adopción de políticas o prácticas intolerantes ante la voluntad de vivir la religión o la espiritualidad en el puesto de trabajo.

Las condiciones de trabajo, el ambiente laboral y la cultura organizativa puede condicionar también el comportamiento de las personas y, en definitiva, su desarrollo. En 1931, Pio XI se hacía eco de la influencia de las condiciones de trabajo de las fábricas de los años 1920, con serios problemas de higiene y de promiscuidad, aparte de prolongados horarios de trabajo que dificultaban la debida atención a la familia. Escribía: "de las fábricas sale ennoblecida la materia inerte, pero los hombres se corrompen y se hacen más viles" (QA 135). Por el contrario, un clima laboral y una cultura que invite a actuar con integridad y fomenten el servicio a los demás pueden facilitar el desarrollo en la empresa.

Un ambiente favorable de respeto a la ley moral natural facilita el desarrollo humano. Hay, en este sentido, una ecología humana que favorece el DHI. Como ha escrito el Papa Francisco,

la ecología humana implica algo muy hondo: la necesaria relación de la vida del ser humano con la ley moral escrita en su propia naturaleza, necesaria para poder crear un ambiente más digno (LS 155).

San Juan Pablo II también exhorta a "salvaguardar las condiciones morales de una auténtica ecología humana" (CA 38) y a prestar la debida atención a una "ecología social" del trabajo (cf. ibídem), aludiendo, sin duda a las condiciones sociales del ámbito laboral que facilitan o desfavorecen el desarrollo de las personas que allí trabajan.

\subsection{Fomentar el DHI}

Fomentar el desarrollo es una exigencia de responsabilidad solidaria con los empleados y otras personas involucradas en la organización. En último término, es también una exigencia de justicia entendida en sentido amplio.

Aparte de la exigencia ética de no impedir el desarrollo humano integral de las personas, existe también la responsabilidad de fomentarlo. Es una exigencia de responsabilidad solidaria con los empleados y otras personas involucradas en la organización. En último término, es también una exigencia de justicia entendida en sentido amplio. 
Más allá de la exigencia moral, promover el desarrollo de empleados y directivos es un modo de favorecer la empresa. En el argot empresarial se ha acuñado conceptos como "capital social" (talentos humanos que contribuyen a mejorar los resultados) y "capital social" (vínculos sociales que disminuyen los costes de coordinación) relacionables con el desarrollo humano. $y$, muchos aceptan hoy que el desarrollo humano de los empleados y directivos es relevante para la empresa, y entienden que "el desarrollo humano puede ayudar a una empresa a gestionar personal, comercializar y vender productos, o negociar el comercio internacional." (Business Dictionary online).

Hay muchos factores de bien común en la empresa que pueden favorecer el desarrollo humano de los empleados. Un liderazgo y una cultura empresarial que fomente la cooperación y el espíritu de servicio es uno de ellos. Otro factor importante para el desarrollo humano en la empresa es la ejemplaridad de los altos directivos, la cual es estimulante para el buen comportamiento de los empleados.

No menos importante para el desarrollo de los empleados en la empresa son el diseño del puesto de trabajo y la organización de la producción. El desarrollo humano se ve favorecido aplicando criterios de participación y subsidiariedad, que permiten aportar los propios talentos en mayor medida en unas estructuras dónde el empleado no sea más que un elemento pasivo (Melé 2005). Con gran clarividencia, Benedicto XVI ha señalado que "la subsidiaridad respeta la dignidad de la persona, en la que ve un sujeto siempre capaz de dar algo a los otros" (CV 57). Y, por tanto, de desarrollarse aportando lo que uno tiene.

La calidad en el trato humano en la empresa puede fomentar el desarrollo, al tiempo que la falta de respeto, la indiferencia y la injusticia pueden dificultarlo. En este sentido procurar el desarrollo se sitúa en el nivel más alto de "calidad humana en el trato" dentro de las organizaciones (Melé 2014).

Una adecuada red de interrelaciones puede también ayudar a desarrollar habilidades y a crecer como persona, empleado, emprendedor y en capacidad para hacer negocios. Como señala Venkatesan (2017), la creación de redes ayuda a establecer amistades fuertes y confiables y conexiones con personas expertas y con una experiencia rica y variada. Actividades complementarias, como el voluntariado empresarial, pueden ayudar a mejorar la sensibilidad ante las necesidades de los demás y desarrollo el sentido de servicio (Wacowska 2014). 


\section{Conclusión}

El desarrollo humano integral es una llamada a cada persona; abarca la vida entera $y$, por tanto, también la vida en la empresa. En este breve ensayo, hemos defendido que el desarrollo humano integral -desarrollo de todo el hombre y de todos los hombres- es posible en la empresa.

Todos quienes forman parte de la empresa están llamados a desarrollarse en su actividad dentro de la organización empresarial. Sin embargo, reconocer esta realidad y más aún favorecer tal desarrollo en la empresa puede parecer extraño para muchas concepciones todavía dominantes en la empresa. Más sorprendente aún puede ser presentar responsabilidades directivas en relación con el del DHI, a menos que se pueda demostrar la contribución del $\mathrm{DHI}$ a la cuenta de resultados, lo cual, por cierto, no es en absoluto inverosímil. Esta visión responde a la idea de que la finalidad de la empresa es obtener ganancias proporcionando bienes y servicios, sin más. Pero, considerar sólo la eficacia productiva y las ganancias es una visión muy pobre de la empresa. La actividad de la empresa afecta a los empleados, y a otros grupos implicados, y este impacto entraña responsabilidad.

La empresa ha de producir y crear riqueza, pero, de acuerdo con la DSI,

la finalidad fundamental de esta producción no es el mero incremento de los productos, ni el beneficio, ni el poder, sino el servicio del hombre, del hombre integral, teniendo en cuanta sus necesidades materiales y sus exigencias intelectuales, morales, espirituales y religiosas (GS 64).

Y, en este "servicio del hombre" ocupan un lugar destacado quienes trabajan en la empresa, los cuales pueden mejorar su desarrollo humano a través de su actividad productiva y en la convivencia empresarial o pueden empeorarlo. Eso es, en primer lugar, responsabilidad de cada persona, pero también afecta a dirigen la empresa y, de otros modo, a quienes forman parte de la comunidad-empresa.

Como hemos tratado de señalar, la responsabilidad de los directivos empresariales en relación con el DHI consiste, en primer lugar, en no impedirlo y, en términos positivos, en favorecerlo; la empresa cuenta con medios para ellos que incluyen la organización, el trato humano, la cultura empresarial y el liderazgo. 


\section{Referencias}

BeNedicto XVI (2009) Encíclica Caritas in veritate. Disponible en www.vatican.va Francisco (2015) Encíclica Laudato si'.

Catecismo de la Iglesia Católica (1997).

Concilio Vaticano II (1965) Constitución Gaudium et spes.

Gleitman, H., Friduund, A. J. y Daniel, R. (2004) Psychology. Nueva York: Norton \& Company.

Hafer, J. y SiRGY, M. J. 1983) "Professional Growth Versus Personal Growth of Salespeople: A General Systems Model", Journal of Personal Selling \& Sales Management 3:2, 22-31.

JUAN PABLO II (1981) Encíclica Laborens exercens.

- (1987) Encíclica Sollicitudo rei socialis.

- (1991) Encíclica Centessimus annus.

LEÓN XIII (1891) Encíclica Rerum novarum.

Maslow, A. H. (1943) "A Theory of Human Motivation", Psychological Review $50,370-96$.

- (1970) Motivation and personality. Nueva York: Harper \& Row, Publishers, Inc.

MeLÉ, D. (2005) "Exploring the Principle of Subsidiarity in Organizational Forms", Journal of Business Ethics 60:3, 293-305.

- (2014) "Human Quality Treatment: Five Organizational Levels", Journal of Business Ethics 120: 457-471.

PABlo VI (1967) Encíclica Populorum progressio. Disponible en www.vatican.va.

Pío XI (1931) Encíclica Quadragesimo anno.

PCJP (Pontificia Comisión Justicia y Paz) (2005) Compendio de Doctrina Social de la lglesia (Madrid/Barcelona: BAC/Planeta). Disponible también en www.vatican.va 
Robitschek, C., Ashton, M. W., Spering, C. C., Geiger, N., Byers, D., Schotts, G. C. y THOEN, M. A. (2012) "Development and Psychometric Evaluation of the Personal Growth Initiative Scale-II", Journal of Counseling Psychology 59:2, 274-87.

Venkatesan, R. (2017) "People Matter: Networking and Career Development", IUP Journal of Soft Skills 11:1, 7-15.

WACOWSKA, A. (2014) "Personal growth and key competences indispensable for professional career development in a knowledge-based-society", Polish Journal of Management Studies 9, 265-74.
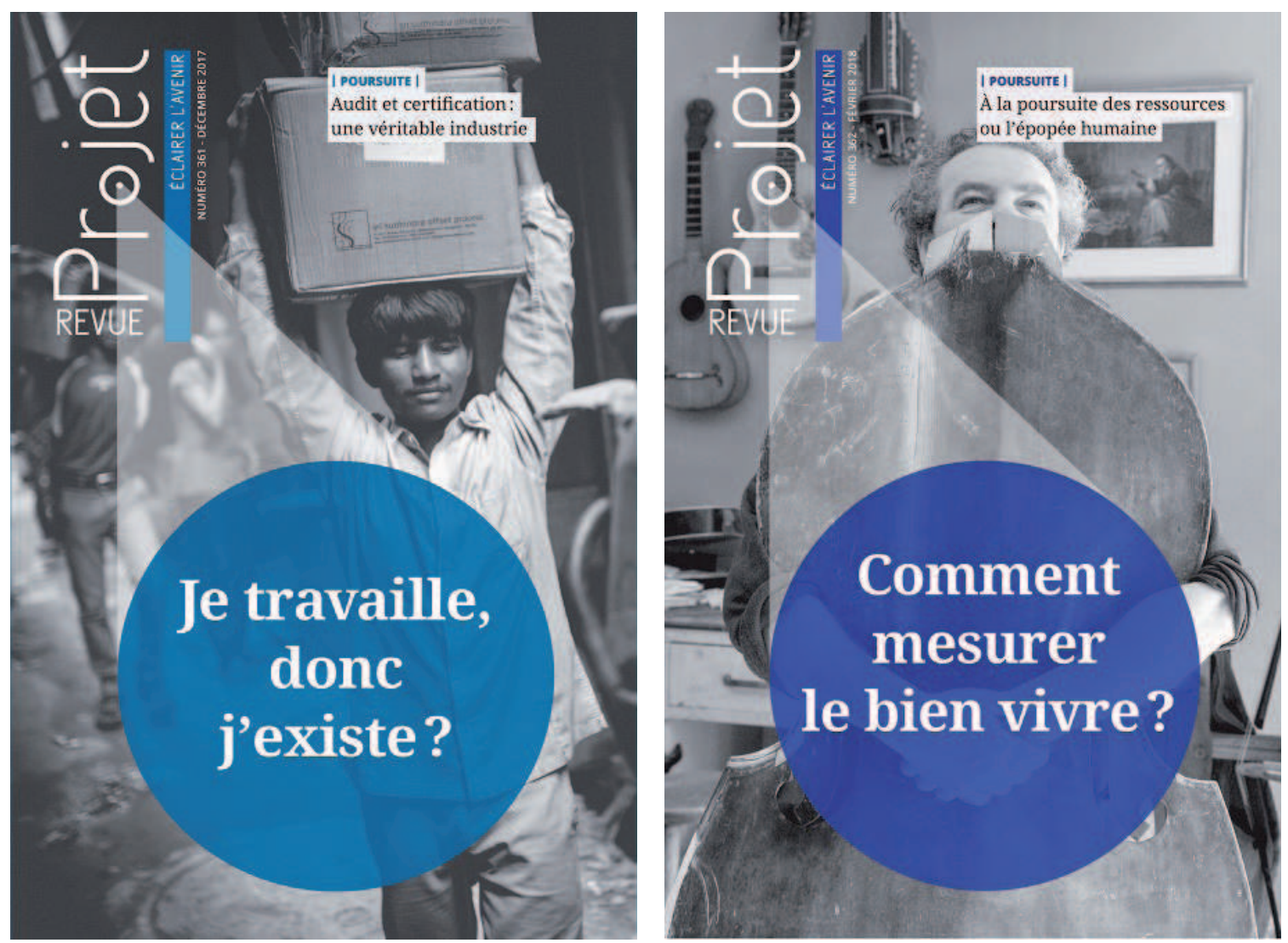